Japan. J. Math.

Vol. 30, No. 1, 2004

\title{
A characterization for pseudo Buchsbaum modules
}

\author{
By Nguyen Tu Cuong and Nguyen Thi Hong LoAN
}

(Received April 15, 2002)

(Revised October 4, 2002)

(from Nagoya Mathematical Journal)

\begin{abstract}
In this paper we study the structure of a new class of modules called pseudo Buchsbaum modules and give a characterization in term of Buchsbaum properties for those modules.
\end{abstract}

\section{Introduction}

Throughout this paper, $R$ denotes a Noetherian local ring with maximal ideal $\mathfrak{m}$ and $M$ a finitely generated $R$-module with $\operatorname{dim} M=d \geq 1$. Let $\underline{x}=\left(x_{1}, \ldots, x_{d}\right)$ be a system of parameters of $M$. We consider the difference between the multiplicity and the length

$$
J_{M}(\underline{x})=e(\underline{x} ; M)-\ell\left(M / Q_{M}(\underline{x})\right),
$$

where $Q_{M}(\underline{x})=\bigcup_{t>0}\left(x_{1}^{t+1}, \ldots, x_{d}^{t+1}\right) M: x_{1}^{t} \cdots x_{d}^{t}$ is a submodule of $M$. It should be mentioned that $J_{M}(\underline{x})$ gives a lot of informations on the structure of $M$. For example, if $M$ is a Cohen-Macaulay module then $Q_{M}(\underline{x})=\left(x_{1}, \ldots, x_{d}\right) M$ by [11]. Therefore $J_{M}(\underline{x})=0$ for all systems of parameters $\underline{x}$ of $M$. Further, it is known by [5] that $\ell\left(M / Q_{M}(\underline{x})\right)$ is just the length of generalized fraction in [14]. Therefore by [14], $\sup _{\underline{x}} J_{M}(\underline{x})<\infty$ if $M$ is a generalized Cohen-Macaulay module. In [3] we also showed that if $M$ is a Buchsbaum module then $J_{M}(\underline{x})$ takes a constant value for every system of parameters $\underline{x}$ of $M$. Unfortunately, the converse of all of the statements above are not true in general. In [8] one studied the structure of modules satisfying $J_{M}(\underline{x})=0$ or $\sup _{\underline{x}} J_{M}(\underline{x})<\infty$. They called it pseudo Cohen-Macaulay module or pseudo generalized Cohen-Macaulay module, respectively. Thus it gives rise to a natural question: what the structure of modules $M$ should be if it fores $J_{M}(\underline{x})$ to take a constant value for all systems of parameters. The purpose of this paper is to study modules which are called pseudo Buchsbaum.

Let $0=\cap N_{i}$ be a reduced primary decomposition of the submodule 0 in $M$,

2000 Mathematics Subject Classification. 13H10, 13D45, $13 \mathrm{C} 99$.

Key words and Phrases. Buchsbaum, pseudo Buchsbaum, local cohomology, multiplicity. 
where $N_{i}$ is $\mathfrak{p}_{i}$-primary. From now on we set

$$
\begin{aligned}
U_{M}(0) & =\bigcap_{\operatorname{dim} R / \mathfrak{p}_{j}=d} N_{j}, \\
\bar{M} & =M / U_{M}(0), \\
\widetilde{M} & =\widehat{M} / U_{\widehat{M}}(0),
\end{aligned}
$$

where $\widehat{M}$ is the $\mathfrak{m}$-adic completion of $M$ and

$$
J(M)=\sum_{i=1}^{d-1}\left(\begin{array}{l}
d-1 \\
i-1
\end{array}\right) \ell\left(H_{\mathfrak{m}}^{i}(M)\right),
$$

where $H_{\mathfrak{m}}^{i}(M)$ stands for the $i^{\text {th }}$ local cohomology module of $M$ with respect to the maximal ideal $\mathfrak{m}$. Note that this invariant $J(M)$ is finite if and only if $M$ is a generalized Cohen-Macaulay module. But it was proved in [8] that $M$ is pseudo Cohen-Macaulay or pseudo generalized Cohen-Macaulay if and only if $\widetilde{M}$ is a Cohen-Macaulay module or a generalized Cohen-Macaulay module over the $\mathfrak{m}$-adic completion $\widehat{R}$ of $R$, respectively. The main result of this paper is to prove the following theorem.

TheOREM 1.1. $M$ is a pseudo Buchsbaum module if and only if $\widetilde{M}$ is a Buchsbaum $\widehat{R}$-module. Moreover, in this case we have

$$
J_{M}(\underline{x})=J(\widetilde{M}),
$$

for every system of parameters $\underline{x}=\left(x_{1}, \ldots, x_{d}\right)$ of $M$.

Note that the Monomial Conjecture posed by M. Hochster (see [13]) says that, $\left(x_{1} \cdots x_{d}\right)^{t} \notin\left(x_{1}^{t+1}, \ldots, x_{d}^{t+1}\right) R$ for every system of parameters $\underline{x}=\left(x_{1}, \ldots, x_{d}\right)$ of the $\operatorname{ring} R(d=\operatorname{dim} R)$ and for all positive integers $t$. It is well-known that the Monomial Conjecture is true for Buchsbaum rings (see [9]). Therefore the following result is an immediate consequence of Theorem 1.1 .

Corollary 1.2. If $R$ is a pseudo Buchsbaum ring, then $R$ satisfies the Monomial Conjecture.

The paper is divided into 4 sections. In Section 2, we shall outline some properties of $J_{M}(\underline{x})$ which will be needed later. Some properties of pseudo Buchsbaum modules will be given in Section 3. In the last section, we shall give the proof of Theorem 1.1 and corollaries. 


\section{The function $J_{M}(\underline{x}(\underline{n}))$}

Let $\underline{x}=\left(x_{1}, \ldots, x_{d}\right)$ be a system of parameters of $M$ and $\underline{n}=\left(n_{1}, \ldots, n_{d}\right)$ a $d$-tuple of positive integers. Set $\underline{x}(\underline{n})=\left(x_{1}^{n_{1}}, \ldots, x_{d}^{n_{d}}\right)$. Then the difference between multiplicities and lengths

$$
J_{M}(\underline{x}(\underline{n}))=n_{1} \cdots n_{d} e(\underline{x} ; M)-\ell\left(M / Q_{M}(\underline{x}(\underline{n}))\right),
$$

can be considered as a function in $\underline{n}$. Note that this function is non-negative ([7, Lemma 3.1]) and ascending, i.e., for $\underline{n}=\left(n_{1}, \ldots, n_{d}\right), \underline{m}=\left(m_{1}, \ldots, m_{d}\right)$ with $n_{i} \geq m_{i}, i=1, \ldots, d, J_{M}(\underline{x}(\underline{n})) \geq J_{M}(\underline{x}(\underline{m}))([3$, Corollary 4.3]).

Moreover, it was proved in [5] that $\ell\left(M / Q_{M}(\underline{x}(\underline{n}))\right)$ is just the length of generalized fraction $M\left(1 /\left(x_{1}^{n_{1}}, \ldots, x_{d}^{n_{d}}, 1\right)\right)$ defined by Sharp and Hamieh [14]. Therefore, we can describe Question 1.2 of [14] as follows: Is $J_{M}(\underline{x}(\underline{n}))$ a polynomial for large enough $\underline{n}$ ( $\underline{n} \gg 0$ for short)? By [3, Lemma 4.1,(ii)], we see that the function $J_{M}(\underline{x}(\underline{n}))$ is bounded above by the polynomial $n_{1} \cdots n_{d} J_{M}(\underline{x})$. More generally, we have the following theorem.

Theorem 2.1 ([7, Theorem 3.2]). The least degree of all polynomials in $\underline{n}$ bounding above the function $J_{M}(\underline{x}(\underline{n}))$ is independent of the choice of the system of parameters $\underline{x}$.

The numerical invariant of $M$ given in the above theorem is called the polynomial type of fractions of $M$ and denoted by $p f(M)$ ([7, Definition 3.3]). For convenience, we stipulate that the degree of the zero-polynomial is equal to $-\infty$.

REMARK 2.2 ([7, Lemma 3.4]). The following statements are true:

(i) Let $\hat{M}$ be the m-adic completion of $M$. Then

$$
p f_{R}(M)=p f_{R}\left(M / H_{\mathfrak{m}}^{0}(M)\right)=p f_{R / \operatorname{Ann}_{R} M}(M)=p f_{\hat{R}}(\hat{M}) ;
$$

(ii) Let $x$ be a parameter element of $M$ with $\operatorname{dim}(0: x)_{M} \leq d-2$. Then

$$
p f(M / x M) \leq p f(M) \leq p f(M / x M)+1 ;
$$

(iii) Suppose that $\operatorname{dim} M=d>1$. Then $p f(M) \leq d-2$.

It was shown in [6] that the function $J_{M}(\underline{x}(\underline{n}))$ is not a polynomial in $\underline{n}$ for $\underline{n} \gg 0$ in general. Therefore the polynomial type of fractions $p f(M)$ plays an important role in the study of the function $J_{M}(\underline{x}(\underline{n}))$. The following definition was introduced in [8, Definition 2.2].

Definition 2.3. (i) $M$ is said to be a pseudo Cohen-Macaulay module if $p f(M)=-\infty$.

(ii) $M$ is said to be a pseudo generalized Cohen-Macaulay module if $p f(M) \leq$ 0. 
By Theorem 2.1, the first statement is equivalent to saying that there exists a system of parameters $\underline{x}=\left(x_{1}, \ldots, x_{d}\right)$ of $M$ such that $J_{M}(\underline{x})=0$ and the second is equivalent to the existence of a system of parameters $\underline{x}=\left(x_{1}, \ldots, x_{d}\right)$ of $M$ such that $J_{M}(\underline{x}(\underline{n}))$ is bounded above by a constant for all $\underline{n} \gg 0$. By Remark 2.2, (i), $M$ is a pseudo Cohen-Macaulay (pseudo generalized Cohen-Macaulay) module if and only if so is the $\mathfrak{m}$-adic completion $\widehat{M}$ of $M$. It should be mentioned that every Cohen-Macaulay module is pseudo Cohen-Macaulay and every generalized Cohen-Macaulay module is pseudo generalized Cohen-Macaulay. Next, we recall some characterizations of these modules. Note that for a module $M$ we often use in this paper the following modules

$$
\bar{M}=M / U_{M}(0), \quad \widetilde{M}=\widehat{M} / U_{\widehat{M}}(0),
$$

where $U_{M}(0)=\bigcap_{\operatorname{dim} R / \mathfrak{p}_{j}=d} N_{j}$ derived from a reduced primary decomposition

$$
0=\bigcap_{\mathfrak{p}_{i} \in \operatorname{Ass}(M)} N_{i}
$$

of the submodule 0 of $M$.

Theorem 2.4 ([8, Theorem 3.1]). Suppose that $R$ admits a dualizing complex. Then the following statements are true.

(i) $M$ is a pseudo Cohen-Macaulay module if and only if $\bar{M}$ is a CohenMacaulay $R$-module.

(ii) $M$ is a pseudo generalized Cohen-Macaulay module if and only if $\bar{M}$ is a generalized Cohen-Macaulay R-module.

Let $\left(x_{1}, \ldots, x_{n}\right)$ be a sequence of elements in $\mathfrak{m}$. Let $\left(y_{1}, \ldots, y_{n}\right)$ be another sequence of $n$ elements such that $\left(y_{1}, \ldots, y_{n}\right) R \subseteq\left(x_{1}, \ldots, x_{n}\right) R$. Then there exists a matrix $B=\left(b_{i j}\right), b_{i j} \in R, 1 \leq i, j \leq n$ such that $y_{i}=\sum_{j=1}^{n} b_{i j} x_{j}$. Put $\delta=\operatorname{det} B$. By [16, Corollary 5.1.15], we have $\delta Q_{M}(\underline{x}) \subseteq Q_{M}(\underline{y})$. Therefore, we obtain a homomorphism

$$
\delta: M / Q_{M}(\underline{x}) \rightarrow M / Q_{M}(\underline{y}),
$$

which is independent of the choice of the matrix $B$. This map $\delta$ is called the determinantal map.

Lemma 2.5 ([3, Lemma 3.1]). Let $\underline{x}=\left(x_{1}, \ldots, x_{d}\right)$ and $\underline{y}=\left(y_{1}, \ldots, y_{d}\right)$ be two systems of parameters of $M$ such that $(\underline{y}) R \subseteq(\underline{x}) R$. Then the determinantal map

$$
\delta: M / Q_{M}(\underline{x}) \longrightarrow M / Q_{M}(\underline{y})
$$

is injective. 
The following properties of $J_{M}(\underline{x})$ were proved in [3].

LEMMA 2.6. The following statements are true.

(i) $\left(\left[3\right.\right.$, Lemma 4.1]) $J_{M}(\underline{x})=J_{\widehat{M}}(\underline{x})=J_{M / H_{\mathrm{m}}^{\circ}(M)}(\underline{x})$, for every system of parameters $\underline{x}$ of $M$.

(ii) $\left(\left[3\right.\right.$, Lemma 4.2]) Let $\underline{x}=\left(x_{1}, \ldots, x_{d}\right)$ and $\underline{y}=\left(x_{1}, \ldots, x_{d-1}, y_{d}\right)$ be two systems of parameters of $M$ such that $(\underline{y}) R \subseteq(\underline{x}) R$. Then $J_{M}(\underline{x}) \leq J_{M}(\underline{y})$.

Let $\underline{x}=\left(x_{1}, \ldots, x_{d}\right)$ be a system of parameters of $M$. Put $M_{1}=M / x_{1} M$, $\underline{x}^{\prime}=\left(x_{2}, \ldots, x_{d}\right)$. For any $(d-1)$-tuple of positive integers $\underline{n}^{\prime}=\left(n_{2}, \ldots, n_{d}\right)$, we set $\underline{x}^{\prime}\left(\underline{n}^{\prime}\right)=\left(x_{2}^{n_{2}}, \ldots, x_{d}^{n_{d}}\right)$ and $\underline{x}\left(\underline{n}^{\prime}\right)=\left(x_{1}, x_{2}^{n_{2}}, \ldots, x_{d}^{n_{d}}\right)$.

LEMmA 2.7. With the same notations as above, if the function $J_{M}(\underline{x}(\underline{n}))$ is constant for all $n_{1}, \ldots, n_{d} \geq 1$ and $\operatorname{dim}\left(0_{\dot{M}} x_{1}\right)<d-1$, so is the function $J_{M_{1}}\left(\underline{x}^{\prime}\left(\underline{n}^{\prime}\right)\right)$.

Proof. Let

$$
\varphi: M_{1} / Q_{M_{1}}\left(\underline{x^{\prime}}\right) \rightarrow M / Q_{M}(\underline{x})
$$

and

$$
\varphi_{\underline{n^{\prime}}}: M_{1} / Q_{M_{1}}\left(\underline{x^{\prime}}\left(\underline{n^{\prime}}\right)\right) \rightarrow M / Q_{M}\left(\underline{x}\left(\underline{n^{\prime}}\right)\right)
$$

be the epimorphisms defined in [3, Lemma 2.2$]$ by

$$
\begin{gathered}
\varphi\left(u_{1}+Q_{M_{1}}\left(\underline{x^{\prime}}\right)\right)=u+Q_{M}(\underline{x}), \\
\varphi_{\underline{n}^{\prime}}\left(u_{1}+Q_{M_{1}}\left(\underline{x^{\prime}}\left(\underline{n^{\prime}}\right)\right)\right)=u+Q_{M}\left(\underline{x}\left(\underline{n^{\prime}}\right)\right)
\end{gathered}
$$

for any $u \in M, u_{1}$ the image of $u$ in $M_{1}$. Then we have the following commutative diagram

$$
\begin{aligned}
& 0 \rightarrow \operatorname{Ker} \varphi \quad \rightarrow \quad M_{1} / Q_{M_{1}}\left(\underline{x^{\prime}}\right) \quad \stackrel{\varphi}{\rightarrow} M / Q_{M}(\underline{x}) \quad \rightarrow 0 \\
& \downarrow \delta^{\prime} \quad \downarrow \delta_{1} \quad \downarrow \delta \\
& 0 \rightarrow \operatorname{Ker} \varphi_{\underline{n^{\prime}}} \rightarrow M_{1} / Q_{M_{1}}\left(\underline{x^{\prime}}\left(\underline{n^{\prime}}\right)\right) \stackrel{\varphi_{n^{\prime}}}{\rightarrow} M / Q_{M}\left(\underline{x}\left(\underline{n^{\prime}}\right)\right) \rightarrow 0,
\end{aligned}
$$

where $\delta, \delta_{1}$ are determinantal maps and the rows are exact sequences. Since $\delta$, $\delta_{1}$ are injective by Lemma 2.5 , the induced homomorphism $\delta^{\prime}$ is injective. Thus $\ell(\operatorname{Ker} \varphi) \leq \ell\left(\operatorname{Ker} \varphi_{\underline{n^{\prime}}}\right)$ for all $n_{2}, \ldots, n_{d} \geq 1$. By the hypothesis there exists a non-negative $k$ such that $J_{M}(\underline{x}(\underline{n}))=k$ for all $n_{1}, \ldots, n_{d} \geq 1$ and $e\left(\underline{x}\left(\underline{n^{\prime}}\right) ; M\right)=$ $e\left(\underline{x^{\prime}}\left(\underline{n^{\prime}}\right) ; M_{1}\right)$. Therefore we get

$$
\begin{gathered}
J_{M_{1}}\left(\underline{x}^{\prime}\right)=J_{M}(\underline{x})-\ell(\operatorname{Ker} \varphi)=k-\ell(\operatorname{Ker} \varphi) \\
J_{M_{1}}\left(\underline{x}^{\prime}\left(\underline{n}^{\prime}\right)\right)=J_{M}\left(\underline{x}\left(\underline{n^{\prime}}\right)\right)-\ell\left(\operatorname{Ker} \varphi_{\underline{n}^{\prime}}\right)=k-\ell\left(\operatorname{Ker} \varphi_{{\underline{n^{\prime}}}^{\prime}}\right) .
\end{gathered}
$$


This implies that $J_{M_{1}}\left(\underline{x^{\prime}}\right) \geq J_{M_{1}}\left(\underline{x^{\prime}}\left(\underline{n^{\prime}}\right)\right)$ for all $n_{2}, \ldots, n_{d} \geq 1$. Since the function $J_{M_{1}}\left(\underline{x^{\prime}}\left(\underline{n^{\prime}}\right)\right)$ is ascending, it follows that $J_{M_{1}}\left(\underline{x^{\prime}}\right)=J_{M_{1}}\left(\underline{x^{\prime}}\left(\underline{n^{\prime}}\right)\right)$ for all $n_{2}, \ldots, n_{d} \geq 1$ and the lemma is proved.

LEMMA 2.8. Let $\underline{x}=\left(x_{1}, \ldots, x_{d}\right)$ and $\underline{y}=\left(y_{1}, \ldots, y_{d}\right)$ be two systems of parameters of $M$ such that $J_{M}(\underline{x}(\underline{n}))=k_{1}$ and $J_{M}(\underline{y}(\underline{n}))=k_{2}$ for all $n_{1}, \ldots, n_{d} \geq$ 1 , where $k_{1}, k_{2} \in \mathbb{N}$. Then we have $k_{1}=k_{2}$.

Proof. Without loss of generality we may assume that $\operatorname{Ann}_{R} M=0$ and $\operatorname{depth} M>0$ by Lemma 2.6, (i). From [16, Proposition 8.2.5], there exists a system of parameters $\underline{z}=\left(z_{1}, \ldots, z_{d}\right)$ of $M$ and positive integers $r_{1}, \ldots, r_{d}$ such that

$$
\begin{aligned}
& \left(x_{1}^{r_{1}}, \ldots, x_{d}^{r_{d}}\right) R \subseteq\left(z_{1}, x_{2}^{r_{2}}, \ldots, x_{d}^{r_{d}}\right) R \subseteq \cdots \subseteq\left(z_{1}, \ldots, z_{d}\right) R \\
& \quad \subseteq\left(z_{1}, \ldots, z_{d-1}, y_{d}\right) R \subseteq \cdots \subseteq\left(z_{1}, y_{2}, \ldots, y_{d}\right) R \subseteq\left(y_{1}, \ldots, y_{d}\right) R .
\end{aligned}
$$

By Lemma 2.6, (ii) we have

$$
\begin{aligned}
& J_{M}\left(x_{1}^{r_{1}}, \ldots, x_{d}^{r_{d}}\right) \geq J_{M}\left(z_{1}, x_{2}^{r_{2}}, \ldots, x_{d}^{r_{d}}\right) \geq \cdots \geq J_{M}(\underline{z}) \\
& \quad \geq J_{M}\left(z_{1}, \ldots, z_{d-1}, y_{d}\right) \geq \cdots \geq J_{M}\left(z_{1}, y_{2}, \ldots, y_{d}\right) \geq J_{M}(\underline{y}) .
\end{aligned}
$$

Hence $k_{1} \geq k_{2}$. Similarly, we get $k_{2} \geq k_{1}$. Therefore $k_{1}=k_{2}$.

The following lemma will be used often in the sequel.

Lemma 2.9. Let $M$ be a pseudo generalized Cohen-Macaulay module and $\underline{x}$ a system of parameters of $M$. Then we have

(i) $J_{M}(\underline{x}(\underline{n}))=J(\widetilde{M})$ for $\underline{n} \gg 0$. Therefore $J_{M}(\underline{z}) \leq J(\widetilde{M})$ holds for every system of parameters $\underline{z}$ of $M$.

(ii) If $R$ admits a dualizing complex then $J_{M}(\underline{x}(\underline{n}))=J(\bar{M})$ for $\underline{n} \gg 0$ and $J_{M}(\underline{z}) \leq J(\bar{M})$ holds for every system of parameters $\underline{z}$ of $M$.

Proof. (i) Suppose that $M$ is a pseudo generalized Cohen-Macaulay module. Then $J_{M}(\underline{x}(\underline{n}))=J_{\widehat{M}}(\underline{x}(\underline{n}))$ for all $n_{1}, \ldots, n_{d} \geq 1$ by Lemma 2.6 , (i). Since the complete ring $\widehat{R}$ always admits a dualizing complex, $\widetilde{M}$ is generalized CohenMacaulay by Theorem 2.4, (ii). Therefore $J(\widetilde{M})<\infty$. Since $\operatorname{dim} U_{\widehat{M}}(0)<d$, there exists a system of parameters $\underline{y}=\left(y_{1}, \ldots, y_{d}\right)$ of $\widehat{M}$ such that $y_{1} U_{\widehat{M}}(0)=0$. It is not difficult to check that $J_{\widehat{M}}(\underline{y}(\underline{n}))=J_{\widetilde{M}}(\underline{y}(\underline{n}))$ for all $n_{1}, \ldots, n_{d} \geq 1$, where $\underline{y}(\underline{n})=\left(y_{1}^{n_{1}}, \ldots, y_{d}^{n_{d}}\right)$. Thus $J_{\widehat{M}}(\underline{y}(\underline{n}))=J_{\widetilde{M}}(\underline{y}(\underline{n}))=J(\widetilde{M})$ for $\underline{n} \gg 0$ by $[14$, Theorem 3.7]. Now the statement follows from Theorem 2.1, Lemma 2.8 and the ascending property of the function $J_{M}(\underline{x}(\underline{n}))$.

(ii) Assume in addition that $R$ admits a dualizing complex. Thus, again by virtue of Theorem 2.4, (ii) $\bar{M}$ is generalized Cohen-Macaulay and therefore $J(\bar{M})<$ $\infty$. Then, by the same method as above we get $J_{M}(\underline{x}(\underline{n}))=J(\bar{M})$, for $\underline{n} \gg 0$ and 
$J_{M}(\underline{z}) \leq J(\bar{M})$ for every system of parameters $\underline{z}$ of $M$.

\section{Pseudo Buchsbaum modules}

We begin with the following definition.

Definition 3.1. An $R$-module $M$ is called a pseudo Buchsbaum module if there exists a constant $K$ such that $J_{M}(\underline{x})=K$ for every system of parameters $\underline{x}$ of $M . R$ is called a pseudo Buchsbaum ring if it is a pseudo Buchsbaum module as a module over itself.

Recall that the notion of standard system of parameters is an important tool for studying generalized Cohen-Macaulay modules. A system of parameters $\underline{x}=$ $\left(x_{1}, \ldots, x_{d}\right)$ of $M$ is called a standard system of parameters if

$$
\ell(M / \underline{x} M)-e(\underline{x} ; M)=\ell\left(M /\left(x_{1}^{2}, \ldots, x_{d}^{2}\right) M\right)-e\left(x_{1}^{2}, \ldots, x_{d}^{2} ; M\right) .
$$

Clearly, if $\underline{x}$ is a standard system of parameters of $M$, so is $\underline{x}(\underline{n})$ for all $n_{1}, \ldots, n_{d} \geq$ 1. $M$ is a generalized Cohen-Macaulay module if and only if $M$ admits a standard system of parameters. Note that standard systems of parameters are also used to characterize Buchsbaum modules (see [15]). A module $M$ is Buchsbaum if and only if every system of parameters of $M$ is standard. The following result will be used often in this paper.

Theorem 3.2 ([3, Theorem 5.1 and Corollary 5.2]). The following statements are true.

(i) Let $M$ be a generalized Cohen-Macaulay module and $\underline{x}=\left(x_{1}, \ldots, x_{d}\right)$ a standard system of parameters of $M$. Then $J_{M}(\underline{x})=J(M)$.

(ii) Let $M$ be a Buchsbaum module. Then $J_{M}(\underline{x})=J(M)$, for any system of parameters $\underline{x}$ of $M$.

The notion of reducing system of parameters was introduced by M. Auslander and D.A. Buchsbaum in [1]. A system of parameters $\underline{x}=\left(x_{1}, \ldots, x_{d}\right)$ is called a reducing system of parameters if $x_{i} \notin \mathfrak{p}$ for all $\mathfrak{p} \in \operatorname{Ass}\left(M /\left(x_{1}, \ldots, x_{i-1}\right) M\right)$ with $\operatorname{dim} R / \mathfrak{p} \geq d-i$ and $i=1, \ldots, d$.

Lemma 3.3. The following statements are true.

(i) $M$ is a pseudo Buchsbaum module if and only if so is $M / H_{\mathfrak{m}}^{0}(M)$.

(ii) Let $M$ be a pseudo Buchsbaum module and $\underline{x}=\left(x_{1}, \ldots, x_{d}\right)$ a reducing system of parameters of $M$. Then $M /\left(x_{1}, \ldots, x_{i}\right) M$ is a pseudo Buchsbaum module for $i=1, \ldots, d$.

Proof. (i) Since $J_{M}(\underline{x})=J_{M / H_{\mathrm{m}}^{\mathrm{o}}(M)}(\underline{x})$ by Lemma 2.6, (i), the statement is clearly true. 
(ii) By induction on $i$, it suffies to show for the case $i=1$. Since $x_{1}$ is a reducing element, $\operatorname{dim}\left(0 ;{ }_{M} x_{1}\right)<d-1$. Therefore the statement follows from Lemma 2.7.

Proposition 3.4. $M$ is a pseudo Buchsbaum module if and only if the $\mathfrak{m}$ adic completion $\widehat{M}$ of $M$ is a pseudo Buchsbaum module over $\widehat{R}$.

Proof. Let $M$ be a pseudo Buchsbaum module. Let $\underline{y}=\left(y_{1}, \ldots, y_{d}\right)$ be a system of parameters of $\widehat{M}$ and $\mathfrak{q}=y \widehat{R} \cap R$. Then there exists a minimal reduction ideal $\mathfrak{b}=\left(x_{1}, \ldots, x_{d}\right) R$ of $\mathfrak{q}$, where $\underline{x}=\left(x_{1}, \ldots, x_{d}\right)$ is a system of parameters of $M$, such that $e(\underline{x} ; M)=e(\mathfrak{q} ; M)$. Thus $e(\underline{x} ; \widehat{M})=e(\underline{y} ; \widehat{M})$. Since $\underline{x} \widehat{M} \subseteq \underline{y} \widehat{M}$, $\ell\left(\widehat{M} / Q_{\widehat{M}}(\underline{x})\right) \geq \ell\left(\widehat{M} / Q_{\widehat{M}}(\underline{y})\right)$ by Lemma 2.5 . Therefore $J_{\widehat{M}}(\underline{x}) \leq J_{\widehat{M}}(\underline{y})$. Since $M$ is a pseudo generalized Cohen-Macaulay module, so is $\widehat{M}$ and hence $J_{\widehat{M}}(\underline{y}) \leq J(\widetilde{M})$ by Lemma 2.9 , (i). On the other hand, since $M$ is pseudo Buchsbaum, $J_{M}(\underline{x})=$ $J(\widetilde{M})$. Thus $J_{\widehat{M}}(\underline{x})=J(\widetilde{M})$ by Lemma 2.6 , (i). Therefore $J_{\widehat{M}}(\underline{y})=J(\widetilde{M})$ and $\widehat{M}$ is a pseudo Buchsbaum module.

Since every system of parameters of $M$ is also a system of parameters of $\widehat{M}$, the converse statement is an immediate consequence of Lemma 2.6, (i).

\section{Proof of Theorem 1.1}

We begin with some auxiliary results as follows.

Lemma 4.1. Let $M$ be a pseudo Buchsbaum module. Suppose that $M$ is generalized Cohen-Macaulay. Then we have $\mathfrak{m} H_{\mathfrak{m}}^{i}(M)=0$ for $i=1, \ldots, d-1$.

Proof. Since $H_{\mathfrak{m}}^{i}\left(M / H_{\mathfrak{m}}^{0}(M)\right) \cong H_{\mathfrak{m}}^{i}(M)$ for all $i>0$, by Lemma 3.3, (i) we may assume without loss of generality that depth $M>0$. We prove the lemma by induction on $d$. For the case $d=1$ there is nothing to prove. Now we assume that $d=2$. Let $x$ be a parameter element of $M$. Then there are by [15, Proposition 1.9, Ch. I] the elements $y_{1}, \ldots, y_{t}$ in $\mathfrak{m}$ such that $\left(y_{1}, \ldots, y_{t}\right)$ is a system of generators of $\mathfrak{m}$ and $y_{i}$ is a parameter element of $M / x M$ for every $i=1, \ldots, t$. Since $M$ is generalized Cohen-Macaulay, the element $y_{i}$ satisfies the hypothesis of Lemma 2.3 in [4] for all $i=1, \ldots, t$. Therefore we obtain by [4, Lemma 2.3] the following exact sequence

$$
0 \rightarrow H_{\mathfrak{m}}^{1}(M) / y_{i} H_{\mathfrak{m}}^{1}(M) \rightarrow M_{i} / Q_{M_{i}}\left(\left(x^{n}\right)\right) \rightarrow M / Q_{M}\left(\left(y_{i}, x^{n}\right)\right) \rightarrow 0
$$

for $n \gg 0$, where $M_{i}:=M / y_{i} M$. Then we have

$$
J_{M}\left(\left(y_{i}, x^{n}\right)\right)=J_{M_{i}}\left(\left(x^{n}\right)\right)+\ell\left(H_{\mathfrak{m}}^{1}(M) / y_{i} H_{\mathfrak{m}}^{1}(M)\right) .
$$

Since $\operatorname{dim} M_{i}=1, J_{M_{i}}\left(\left(x^{n}\right)\right)=0$ by [14, Proposition 3.1]. Thus $J_{M}\left(\left(y_{i}, x^{n}\right)\right)=$ $\ell\left(H_{\mathfrak{m}}^{1}(M) / y_{i} H_{\mathfrak{m}}^{1}(M)\right)$. On the other hand, for $n \gg 0 J_{M}\left(\left(y_{i}^{n}, x^{n}\right)\right)=\ell\left(H_{\mathfrak{m}}^{1}(M)\right)$ 
by [14, Theorem 3.7]. Therefore $\ell\left(H_{\mathfrak{m}}^{1}(M) / y_{i} H_{\mathfrak{m}}^{1}(M)\right)=\ell\left(H_{\mathfrak{m}}^{1}(M)\right)$ since $M$ is a pseudo Buchsbaum module. This implies that $y_{i} H_{\mathfrak{m}}^{1}(M)=0$ for all $i=1, \ldots, t$. Thus $\mathfrak{m} H_{\mathfrak{m}}^{1}(M)=0$.

Let $d \geq 3$. Since $M$ is a generalized Cohen-Macaulay module and depth $M>0$, there always exists a non-zero divisor $x \in \mathfrak{m}$ such that $x H_{\mathfrak{m}}^{i}(M)=0$, $i=0, \ldots, d-1$. From the exact sequence

$$
0 \rightarrow M \stackrel{x}{\rightarrow} M \rightarrow M / x M \rightarrow 0
$$

we get the following short exact sequence of local cohomology modules

$$
0 \rightarrow H_{\mathfrak{m}}^{i}(M) \rightarrow H_{\mathfrak{m}}^{i}(M / x M) \rightarrow H_{\mathfrak{m}}^{i+1}(M) \rightarrow 0,
$$

for $i=1, \ldots, d-2$. Since $M / x M$ is a generalized Cohen-Macaulay module and a pseudo Buchsbaum module by Lemma 3.3, (ii), we can apply the inductive hypothesis for $M / x M$ to obtain that $\mathfrak{m} H_{\mathfrak{m}}^{i}(M / x M)=0$ for $i=1, \ldots, d-2$. Therefore $\mathfrak{m} H_{\mathfrak{m}}^{i}(M)=0$ for $i=1, \ldots, d-1$.

Recall that $M$ is called a quasi-Buchsbaum module if $\mathfrak{m} H_{\mathfrak{m}}^{i}(M)=0$, for all $i \neq d$. Hence Lemma 4 .1 leads immediately to the following consequence.

Corollary 4.2. Let $M$ be a pseudo Buchsbaum module. Suppose that $M$ is generalized Cohen-Macaulay with $\mathfrak{m} H_{\mathfrak{m}}^{0}(M)=0$. Then $M$ is a quasi-Buchsbaum module.

Let $0=\cap N_{i}$ be a reduced primary decomposition of the submodule 0 in $M$, where $N_{i}$ is $\mathfrak{p}_{i}$-primary. Note that $U_{M}(0)=\bigcap_{\operatorname{dim} R / \mathfrak{p}_{j}=d} N_{j}=H_{\mathfrak{m}}^{0}(M)$ when $M$ is a generalized Cohen-Macaulay module. Thus the following result is a special case of Theorem 1.1.

Lemma 4.3. The following statments are equivalent:

(i) $M / H_{\mathfrak{m}}^{0}(M)$ is a Buchsbaum module.

(ii) $M$ is generalized Cohen-Macaulay and pseudo Buchsbaum.

Proof. (i) $\Rightarrow$ (ii) is clear by Theorem 3.2, (ii) and Lemma 3.3, (i).

(ii) $\Rightarrow$ (i): By virtue of Lemma 3.3, (i), we may assume without loss of generality that $\operatorname{depth} M>0$. We show the lemma by induction on $d$. It is trivial for the case $d=1$. Suppose $d \geq 2$. Let $x$ be any parameter element of $M$. Then $x$ is a non-zero divisor of $M$. Put $M^{\prime}=M / x M$. Then $\operatorname{dim} M^{\prime}=d-1$ and $M^{\prime}$ satisfies the condition (ii). It follows from the inductive hypothesis that $M^{\prime} / H_{\mathfrak{m}}^{0}\left(M^{\prime}\right)$ is a Buchsbaum module. Let

$$
x M:\langle\mathfrak{m}\rangle=\left\{u \in M \mid \exists n>0, \mathfrak{m}^{n} \cdot u \subseteq x M\right\} .
$$

Then $M / x M:\langle\mathfrak{m}\rangle$ is a Buchsbaum module since

$$
M / x M:\langle\mathfrak{m}\rangle \cong M^{\prime} / H_{\mathfrak{m}}^{0}\left(M^{\prime}\right)
$$


On the other hand $\mathfrak{m} H_{\mathfrak{m}}^{1}(M)=0$ by Lemma 4.1. Therefore $M$ is a Buchsbaum module by [15, Proposition 2.23, Ch. I] and the lemma is proved.

Proof of TheOREM 1.1. Without any loss of generality, we may assume by Proposition 3.4 that $R=\widehat{R}$. Since $\widehat{R}$ always admits a dualizing complex, Theorem 1.1 is now an immediate consequence of the following lemma.

Lemma 4.4. Suppose that $R$ admits a dualizing complex. Then $M$ is a pseudo Buchsbaum module if and only if $\bar{M}=M / U_{M}(0)$ is a Buchsbaum $R$-module. Moreover, in this case we have

$$
J_{M}(\underline{x})=J(\bar{M})
$$

for every system of parameters $\underline{x}=\left(x_{1}, \ldots, x_{d}\right)$ of $M$.

Proof. The last statement of the lemma follows immediately from Lemma 2.9 , (ii). So we need only to prove the first one.

a) Sufficient condition. Assume that $\bar{M}$ is a Buchsbaum module. The statement is trivial for the case $d=1$. Suppose now that $d>1$. First we prove the following claim.

Claim. For any system of parameters $\underline{x}$ of $M$ and $t \gg 0$,

$$
\left(x_{1}^{t+1}, \ldots, x_{d}^{t+1}\right) M: x_{1}^{t} \cdots x_{d}^{t}=\left[\left(x_{1}^{t+1}, \ldots, x_{d}^{t+1}\right) M+U_{M}(0)\right]: x_{1}^{t} \cdots x_{d}^{t}
$$

Proof of Claim. It is clear that the inclusion $\subseteq$ is true for all $t$. So we only need to prove the converse. Note that for $t \gg 0$ the left submodule in the equality of the claim is just $Q_{M}(\underline{x})$. Let

$$
a \in\left[\left(x_{1}^{t+1}, \ldots, x_{d}^{t+1}\right) M+U_{M}(0)\right]: x_{1}^{t} \cdots x_{d}^{t} .
$$

Since $\bar{M}$ is a Buchsbaum module, we get by [10, Theorem 2.3] that $\left[\left(x_{1}^{t+1}, \ldots, x_{d}^{t+1}\right) M+U_{M}(0)\right]: x_{1}^{t} \cdots x_{d}^{t}=\sum_{i=1}^{d}\left[\left(x_{1}, \ldots, \hat{x}_{i}, \ldots, x_{d}\right) M+U_{M}(0)\right]: x_{i}$

for all $t \geq 1$, where $\wedge$ means deleting the item. Therefore, there are $a_{1}, \ldots, a_{d}$ in $M$ with $a_{i} x_{i} \in\left[\left(x_{1}, \ldots, \hat{x}_{i}, \ldots, x_{d}\right) M+U_{M}(0)\right]$ such that $a=\sum_{i=1}^{d} a_{i}$.

For any positive integers $n_{1}, \ldots, n_{d} \geq 1$, we have

$$
\begin{aligned}
a_{i} x_{i} \prod_{i=1}^{d} x_{i}^{n_{i}-1} & \in \prod_{i=1}^{d} x_{i}^{n_{i}-1}\left[\left(x_{1}, \ldots, \hat{x}_{i}, \ldots, x_{d}\right) M+U_{M}(0)\right] \\
& \subseteq\left(x_{1}^{n_{1}}, \ldots, \hat{x}_{i}^{n_{i}}, \ldots, x_{d}^{n_{d}}\right) M+U_{M}(0) .
\end{aligned}
$$


Hence

$$
a \prod_{i=1}^{d} x_{i}^{n_{i}-1} \in \sum_{i=1}^{d}\left[\left(x_{1}^{n_{1}}, \ldots, \hat{x}_{i}^{n_{i}}, \ldots, x_{d}^{n_{d}}\right) M+U_{M}(0)\right]: x_{i} .
$$

On the other hand, $M$ is a pseudo generalized Cohen-Macaulay module by Theorem 2.4, (ii). So using Lemma 2.9 , (ii) we get $J_{M}(\underline{x}(\underline{n}))=J(\bar{M})$ for $\underline{n} \gg 0$. It follows that $J_{M}(\underline{x}(\underline{n}))=J_{\bar{M}}(\underline{x}(\underline{n}))$, by Theorem 3.2 , (ii) for $\underline{n} \gg 0$. Since $e(\underline{x} ; M)=$ $e(\underline{x} ; \bar{M})$, it implies that $\ell\left(M / Q_{M}(\underline{x}(\underline{n}))\right)=\ell\left(\bar{M} / Q_{\bar{M}}(\underline{x}(\underline{n}))\right)$ for $\underline{n} \gg 0$. Therefore the epimorphism $M / Q_{M}(\underline{x}(\underline{n})) \rightarrow \bar{M} / Q_{\bar{M}}(\underline{x}(\underline{n}))$ defined by $\varphi\left(u+Q_{M}(\underline{x}(\underline{n}))\right)=$ $\bar{u}+Q_{\bar{M}}(\underline{x}(\underline{n}))$ for any $u \in M$ with $\bar{u}$ being the image of $u$ in $\bar{M}$ is an isomorphism for $\underline{n} \gg 0$. Thus we have

$$
\begin{aligned}
& \left(x_{1}^{n_{1}(k+1)}, \ldots, x_{d}^{n_{d}(k+1)}\right) M: \prod_{i=1}^{d} x_{i}^{n_{i} k} \\
& \quad=\left[\left(x_{1}^{n_{1}(k+1)}, \ldots, x_{d}^{n_{d}(k+1)}\right) M+U_{M}(0)\right]: \prod_{i=1}^{d} x_{i}^{n_{i} k}
\end{aligned}
$$

for $\underline{n} \gg 0$ and $k \gg 0$. Further, since $\bar{M}$ is a Buchsbaum module, the right term of the equality above is just equal by [10, Theorem 2.3$]$ to

$$
\sum_{i=1}^{d}\left[\left(x_{1}^{n_{1}}, \ldots, x_{i}^{n_{i}}, \ldots, x_{d}^{n_{d}}\right) M+U_{M}(0)\right]: x_{i}
$$

for all $n_{1}, \ldots, n_{d} \geq 1$ and $k \geq 1$. Therefore we obtain

$$
\left(x_{1}^{n_{1}(k+1)}, \ldots, x_{d}^{n_{d}(k+1)}\right) M: \prod_{i=1}^{d} x_{i}^{n_{i} k}=\sum_{i=1}^{d}\left[\left(x_{1}^{n_{1}}, \ldots, \hat{x}_{i}^{n_{i}}, \ldots, x_{d}^{n_{d}}\right) M+U_{M}(0)\right]: x_{i},
$$

for $\underline{n} \gg 0$ and $k \gg 0$. Combining these facts we have

$$
a \in\left(x_{1}^{n_{1} k+n_{1}}, \ldots, x_{d}^{n_{d} k+n_{d}}\right) M: \prod_{i=1}^{d} x_{i}^{n_{i} k+n_{i}-1}
$$

for $\underline{n} \gg 0$ and $k \gg 0$. Thus

$$
a \in\left(x_{1}^{(t+1)}, \ldots, x_{d}^{(t+1)}\right) M: x_{1}^{t} \cdots x_{d}^{t}
$$

for $t \gg 0$. This finishes the proof of the claim.

Now we continue to prove the sufficient condition of Lemma 4.4. Let $\underline{x}$ be any system of parameters of $M$. It follows by Claim that $M / Q_{M}(\underline{x}) \cong \bar{M} / Q_{\bar{M}}(\underline{x})$. Therefore $J_{M}(\underline{x})=J_{\bar{M}}(\underline{x})$. But $\bar{M}$ is a Buchsbaum module by the hypothesis, $J_{\bar{M}}(\underline{x})=J(\bar{M})$. Therefore $M$ is a pseudo Buchsbaum module. 
In order to prove the necessary condition of Lemma 4.4 we need the following lemma.

Lemma 4.5. Let $\bar{M}$ be a generalized Cohen-Macaulay module, $y=$ $\left(y_{1}, \ldots, y_{d}\right)$ a system of parameters of $\bar{M}$, and $i$ a positive integer, $i \leq d$. Then there exists a part of a system of parameters $\left(x_{1}, \ldots, x_{i}\right)$ of $M$ such that $x_{j}-y_{j} \in \operatorname{Ann}_{R} \bar{M}$ for all $j=1, \ldots, i$.

Proof. We prove this result by induction on $i$. Let $i=1$, we choose $x_{1}=y_{1}$. Suppose we already have for $i \geq 1$ a part of the system of parameters $x_{1}, \ldots, x_{i}$ for $M$ such that $x_{j}-y_{j} \in \operatorname{Ann}_{R} \bar{M}$ for $j=1, \ldots, i$. We have to show that there exists a parameter element $x_{i+1}$ of $M_{i}$ with $x_{i+1}-y_{i+1} \in \operatorname{Ann}_{R} \bar{M}$, where $M_{i}=M /\left(x_{1}, \ldots, x_{i}\right) M$.

Set

$$
T=\left\{\mathfrak{p} \mid \mathfrak{p} \in \operatorname{Assh}\left(M_{i}\right), y_{i+1} \notin \mathfrak{p}\right\}, \quad L=\operatorname{Assh} M_{i} \backslash T,
$$

where for a module $N$ we denote

$$
\operatorname{Assh} N=\{\mathfrak{p} \in \operatorname{Ass} N \mid \operatorname{dim} R / \mathfrak{p}=\operatorname{dim} N\} .
$$

If $L=\emptyset$, we choose $x_{i+1}=y_{i+1}$ and the sequence $x_{1}, \ldots, x_{i+1}$ satisfies the required condition of the lemma. Suppose that $L \neq \emptyset$. Note that

$$
\operatorname{Assh} \bar{M} /\left(x_{1}, \ldots, x_{i}\right) \bar{M} \subseteq \operatorname{Assh} M_{i}=T \cup L .
$$

Since

$$
\operatorname{Assh} \bar{M} /\left(y_{1}, \ldots, y_{i}\right) \bar{M}=\operatorname{Assh} \bar{M} /\left(x_{1}, \ldots, x_{i}\right) \bar{M}
$$

by the choice of $x_{1}, \ldots, x_{i}$, it follows that

$$
\operatorname{Assh} \bar{M} /\left(x_{1}, \ldots, x_{i}\right) \bar{M} \subseteq T .
$$

Let $z \in \mathfrak{m}$ be such that

$$
z \in\left(\bigcap_{\mathfrak{p} \in T} \mathfrak{p}\right) \backslash\left(\bigcup_{\mathfrak{q} \in L} \mathfrak{q}\right)
$$

Since $\bar{M} /\left(x_{1}, \ldots, x_{i}\right) \bar{M}$ is a generalized Cohen-Macaulay module, we get

$$
\begin{aligned}
z \in \bigcap_{\mathfrak{p} \in T} \mathfrak{p} \subseteq \bigcap_{\mathfrak{p} \in \operatorname{Assh}} \bigcap_{\bar{M} /\left(x_{1}, \ldots, x_{i}\right) \bar{M}} \mathfrak{p} & =\operatorname{Rad}\left(\operatorname{Ann}_{R} \bar{M} /\left(x_{1}, \ldots, x_{i}\right) \bar{M}\right) \\
& =\operatorname{Rad}\left(\operatorname{Ann}_{R} \bar{M}+\left(x_{1}, \ldots, x_{i}\right) R\right) .
\end{aligned}
$$

Therefore there exist a positive integer $k$ and elements $a_{i+1} \in \operatorname{Ann}_{R} \bar{M}, r_{1}, \ldots, r_{i} \in$ $R$ such that $z^{k}=a_{i+1}+\sum_{j=1}^{i} r_{j} x_{j}$. Since $z^{k} \in\left(\bigcap_{\mathfrak{p} \in T} \mathfrak{p}\right) \backslash\left(\bigcup_{\mathfrak{q} \in L} \mathfrak{q}\right)$ and $\left(x_{1}, \ldots, x_{i}\right) R \subseteq \mathfrak{p}$ for all $\mathfrak{p} \in \operatorname{Assh} M_{i}, a_{i+1} \in \bigcap_{\mathfrak{p} \in T} \mathfrak{p}$ and $a_{i+1} \notin \bigcup_{\mathfrak{q} \in L} \mathfrak{q}$. We 
choose now $x_{i+1}=y_{i+1}+a_{i+1}$. Then $x_{i+1}$ is a parameter element of $M_{i}$ and the lemma is therefore proved.

b) Necessary condition of Lemma 4.4. Assume that $M$ is a pseudo Buchsbaum module. Then $\bar{M}$ is a generalized Cohen-Macaulay module by Theorem 2.4, (ii). Let $y=\left(y_{1}, \ldots, y_{d}\right)$ be any system of parameters of $\bar{M}$. Then there exists by Lemma 4.5 a system of parameters $\underline{x}=\left(x_{1}, \ldots, x_{d}\right)$ of $M$ such that $e(\underline{y} ; \bar{M})=e(\underline{x} ; \bar{M})$ and $\ell\left(\bar{M} / Q_{\bar{M}}(\underline{y})\right)=\ell\left(\bar{M} / Q_{\bar{M}}(\underline{x})\right)$. Therefore

$$
J(\bar{M}) \geq J_{\bar{M}}(\underline{y})=J_{\bar{M}}(\underline{x}) .
$$

Since the homomorphism $\varphi: M / Q_{M}(\underline{x}) \rightarrow \bar{M} / Q_{\bar{M}}(\underline{x})$ is surjective, $\ell\left(\bar{M} / Q_{\bar{M}}(\underline{x})\right) \leq$ $\ell\left(M / Q_{M}(\underline{x})\right)$. Hence

$$
J(\bar{M})=J_{M}(\underline{x}) \leq J_{\bar{M}}(\underline{x})
$$

by Lemma 2.9,(ii). Therefore we get

$$
J_{\bar{M}}(\underline{x})=J(\bar{M})=J_{\bar{M}}(\underline{y}) .
$$

So $\bar{M}$ is pseudo Buchsbaum. Moreover, since depth $\bar{M}>0$, it follows that $\bar{M}$ is a Buchsbaum module by Lemma 4.3. This finishes the proof of Lemma 4.4 and therefore the proof of Theorem 1.1 is complete.

Proof of Corollary 1.2. Suppose that $R$ is a pseudo Buchsbaum ring. Then $\widetilde{R}=\widehat{R} / U_{\widehat{R}}(0)$ is a Buchsbaum $\widehat{R}$-module by Theorem 1.1. Therefore $\widetilde{R}$ is a Buchsbaum ring. Since the Monomial Conjecture holds true for Buchsbaum rings by [9], $\ell\left(\hat{R} / Q_{\hat{R}}(\underline{x})\right) \neq 0$. Therefore $\ell\left(R / Q_{R}(\underline{x})\right) \neq 0$ and the corollary is proved.

For the next consequence of Theorem 1.1 we need to recall the polynomial type of a module defined in [2] as follows.

Set

$$
I_{M}(\underline{x}(\underline{n}))=\ell\left(M /\left(x_{1}^{n_{1}}, \ldots, x_{d}^{n_{d}}\right) M\right)-n_{1} \cdots n_{d} e(\underline{x} ; M) .
$$

It was proved in [2] that the least degree of all polynomials in $\underline{n}$ bounding above the function $I_{M}(\underline{x}(\underline{n}))$ does not depend on the choice of $\underline{x}$. This least degree is called the polynomial type of $M$ and denoted by $p(M)$. Then $M$ is Cohen-Macaulay if and only if $p(M)=-\infty$ and $M$ is generalized Cohen-Macaulay if and only if $p(M) \leq 0$. In general, we have $p f(M) \leq p(M) \leq d-1$ by [7].

Corollary 4.6. Let $M$ be a pseudo Buchsbaum module. Then we have $\mathfrak{m} H_{\mathfrak{m}}^{i}(M)=0$ for $i=p(M)+1, \ldots, d-1$, where $p(M)$ is the polynomial type of the module $M$. 
Proof. The case $p(M) \leq 0$ is proved by Lemma 4.1. Assume that $p(M)>$ 0 . Since $M$ is pseudo Buchsbaum if and only if so is $\hat{M}$ by Proposition 3.4 and $p(M)=p(\hat{M})$ by [2], we may assume without loss of generality that $R$ is complete. Since $M$ is pseudo Buchsbaum, $\ell\left(H_{\mathfrak{m}}^{i}(M)\right)<\infty$ for $i=p(M)+1, \ldots, d-1$ by $[7$, Lemma 3.6,(i)]. Therefore $\operatorname{dim} U_{M}(0) \leq p(M)$ by [2, Theorem 3.1]. On the other hand, from the exact sequence

$$
0 \rightarrow U_{M}(0) \rightarrow M \rightarrow \bar{M} \rightarrow 0
$$

we get $H_{\mathfrak{m}}^{i}(M) \cong H_{\mathfrak{m}}^{i}(\bar{M})$ for $i=p(M)+1, \ldots, d-1$. So the statement follows from Theorem 1.1.

We see by Theorem 1.1 that the class of pseudo Buchsbaum modules contains the class of Buchsbaum modules. Here we give some examples to show that there exist pseudo Buchsbaum modules which are not Buchsbaum modules, not even generalized Cohen-Macaulay modules. Moreover, there exist generalized CohenMacaulay modules which are not pseudo Buchsbaum modules.

Example. (1) Let $k\left[\left[X_{1}, \ldots, X_{4}\right]\right]$ be the formal power series ring. Put

$$
A=k\left[\left[X_{1}, \ldots, X_{4}\right]\right] /\left(X_{1}, X_{2}\right) \cap\left(X_{3}, X_{4}\right) \cap\left(X_{1}^{2}, X_{2}, X_{3}\right) .
$$

Then

$$
A / U_{A}(0)=k\left[\left[X_{1}, \ldots, X_{4}\right]\right] /\left(X_{1}, X_{2}\right) \cap\left(X_{3}, X_{4}\right) .
$$

It is clear that, $A$ is not a generalized Cohen-Macaulay ring. Since $A / U_{A}(0)$ is a Buchsbaum ring (see [15, Example 6, Introduction]), $A$ is a pseudo-Buchsbaum ring by Theorem 1.1. Moreover, it is easy to see that $J_{A}(\underline{x})=1$ for every $\underline{x}$ of $A$.

(2) Let $R=k\left[\left[X_{1}, \ldots, X_{n}\right]\right](n \geq 2)$ be the formal power series ring and $M=\left(X_{1}^{2}, X_{2}, \ldots, X_{n}\right) R$. From the exact sequence

$$
0 \rightarrow M \rightarrow R \rightarrow R /\left(X_{1}^{2}, X_{2}, \ldots, X_{n}\right) R \rightarrow 0,
$$

we have $H_{\mathfrak{m}}^{i}(M)=0$, for $i \neq 1, n$ and $H_{\mathfrak{m}}^{1}(M) \cong R /\left(X_{1}^{2}, X_{2}, \ldots, X_{n}\right) R$. Therefore $M$ is a generalized Cohen-Macaulay module. On the other hand, as $\mathfrak{m} H_{\mathfrak{m}}^{1}(M) \neq$ $0, M$ is not a Buchsbaum module. Moreover, $U_{M}(0)=0$, hence $M / U_{M}(0)$ is not a Buchsbaum module which implies that $M$ is not a pseudo-Buchsbaum module.

(3) Let $M=\bigoplus_{i=1}^{n} M_{i}$, where $M_{i}$ is a $R$-module which $\operatorname{dim} M_{i}=\operatorname{dim} M$ for $i=1, \ldots, n$. Then $M$ is a pseudo Buchsbaum module if $M_{i}$ is so for every $i=1, \ldots, n$. Indeed, it suffices to prove this for $n=2$. Clearly, $\underline{x}=\left(x_{1}, \ldots x_{d}\right)$ is a system of parameters of $M$ if and only if it is a system of parameters of $M_{1}$ and $M_{2}$. For any set of positive integers $\underline{n}=\left(n_{1}, \ldots, n_{d}\right)$, it is clear that

$$
e(\underline{x}(\underline{n}) ; M)=e\left(\underline{x}(\underline{n}) ; M_{1}\right)+e\left(\underline{x}(\underline{n}) ; M_{2}\right),
$$




$$
Q_{M}(\underline{x}(\underline{n}))=Q_{M_{1}}(\underline{x}(\underline{n})) \oplus Q_{M_{2}}(\underline{x}(\underline{n}))
$$

Therefore

$$
J_{M}(\underline{x}(\underline{n}))=J_{M_{1}}(\underline{x}(\underline{n}))+J_{M_{2}}(\underline{x}(\underline{n})) .
$$

It follows that $M$ is a pseudo Buchsbaum module if $M_{1}$ and $M_{2}$ are pseudo Buchsbaum modules.

Now we consider the Matlis dual $D^{d}(M)=\operatorname{Hom}_{R}\left(H_{\mathfrak{m}}^{d}(M), E\right)$ of the $d^{\text {th }}$-local cohomology module $H_{\mathrm{m}}^{d}(M)$, where $E$ denotes the injective hull of the residue field $R / \mathfrak{m}$ of $R$. It is known that $D^{d}(M)$ is a finitely generated $\widehat{R}$-module. Moreover, if $M$ has a canonical module then (see [12])

$$
K_{\widehat{M}} \cong K_{M} \otimes_{R} \widehat{R} \cong D^{d}(M)
$$

as $\widehat{R}$-module. Next, we are interested in the Buchsbaum property for the module $D^{d}(M)$ of a pseudo Buchsbaum module.

Corollary 4.7. Let $M$ be a pseudo Buchsbaum module. Then $D^{d}(M)$ is a Buchsbaum $\widehat{R}$-module.

Proof. Since $\operatorname{dim} U_{\widehat{M}}(0) \leq d-1$, we get from the exact sequence

$$
0 \rightarrow U_{\widehat{M}}(0) \rightarrow \widehat{M} \rightarrow \widetilde{M} \rightarrow 0
$$

an isomorphism $H_{\widehat{\mathfrak{m}}}^{d}(\widehat{M}) \cong H_{\widehat{\mathfrak{m}}}^{d}(\widetilde{M})$. Therefore $K_{\widehat{M}} \cong K_{\widetilde{M}}$ as $\widehat{R}$-module. On the other hand, since $\widehat{M}$ is a Buchsbaum $\widehat{R}$-module by Theorem $1.1, K_{\widehat{M}} \cong D^{d}(M)$ is a Buchsbaum $\widehat{R}$-module by [15, Theorem 4.9 , Ch. II].

The following corollary follows immediately from the corollary above.

Corollary 4.8. Let $M$ be a pseudo Buchsbaum module having a canonical module $K_{M}$. Then $K_{M}$ is a Buchsbaum module.

We denote by $\mu_{R}(M)$ the minimal number of generators of $M$. The multiplicity of $M$ with respect to the maximal ideal $\mathfrak{m}$ is denoted by $e(M)$. It is well known that for any finitely generated $R$-module we have

$$
\mu_{R}(M) \leq e(M)+I(M),
$$

where $I(M)=\sum_{i=0}^{d-1}\left(\begin{array}{c}d-1 \\ i\end{array}\right) \ell\left(H_{\mathfrak{m}}^{i}(M)\right)$. Then $M$ is called a Linear Maximal Buchsbaum module (see [17]) when $\mu_{R}(M)=e(M)+I(M)$.

Let $R$ be a pseudo Buchsbaum ring. Then we have by Theorem 1.1 that

$$
J_{R}(\underline{x})=J(\widetilde{R})=\sum_{i=1}^{d-1}\left(\begin{array}{l}
d-1 \\
i-1
\end{array}\right) \ell\left(H_{\mathfrak{m}}^{i}(\widetilde{R})\right),
$$


for any system of parameters $\underline{x}=\left(x_{1}, \ldots, x_{d}\right)$ of $R$, where $\widetilde{R}=\widehat{R} / U_{\widehat{R}}(0)$ and $d=$ $\operatorname{dim} R$. Furthermore, since $R$ satisfies the Monomial Conjecture by Corollary 1.2 , $e(\underline{x} ; R) \geq 1+J(\widetilde{R})$, for any system of parameters $\underline{x}$ of $R$. It follows that $e(R) \geq$ $1+J(\widetilde{R})$. When $e(R)=1+J(\widetilde{R})$, we say that $R$ has the minimal multiplicity.

Corollary 4.9. Let $R$ be a pseudo Buchsbaum ring. Then the following statments are equivalent:

(i) $R$ has the minimal multiplicity.

(ii) $D^{d}(R)$ is a Linear Maximal Buchsbaum $\widehat{R}$-module.

Proof. (i) $\Rightarrow($ ii): $\quad$ Since $R$ has the minimal multiplicity, $1+J(\widetilde{R})=e(R)=$ $e(\widetilde{R})$. Thus $\widetilde{R}$ is by Theorem 1.1 a Buchsbaum ring with the minimal multiplicity. Then we can show as in the proof of Corollary 4.7 that

$$
K_{\widetilde{R}} \cong K_{\widehat{R}} \cong D^{d}(R)
$$

is a Linear Maximal Buchsbaum $\widehat{R}$-module by [17, Proposition 3.1].

(ii) $\Rightarrow$ (i): First we have as above that $K_{\widetilde{R}} \cong K_{\widehat{R}} \cong D^{d}(R)$ is a Linear Maximal Buchsbaum $\widehat{R}$-module. Hence $\widetilde{R}$ is a Buchsbaum ring with the minimal multiplicity by [17, Proposition 3.1]. Therefore $R$ has the minimal multiplicity.

Let now $M$ be a pseudo Buchsbaum module, then $J_{M}(\underline{x})=J(\widetilde{M})$ for all system of parameters $\underline{x}$ of $M$. Hence $e(\underline{x} ; M) \geq J(\widetilde{M})$ for all system of parameters of $M$. Therefore $e(M) \geq J(\widetilde{M})$. In the case the equality holds, we get the following result.

Corollary 4.10. Suppose that $\operatorname{dim} M=\operatorname{dim} R \geq 2$. Then the following statments are equivalent:

(i) $M$ is a pseudo Buchsbaum module with $e(M)=J(\widetilde{M})$.

(ii) $\widetilde{M}$ is a Linear Maximal Buchsbaum $\widehat{R}$-module and $\mu_{\widehat{R}}(\widetilde{M})=$ $\sum_{i=0}^{d-1}\left(\begin{array}{c}d \\ i\end{array}\right) \ell\left(H_{\widehat{\mathrm{m}}}^{i}(\widetilde{M})\right)$.

Proof. (i) $\Rightarrow$ (ii): Suppose that $\underline{x}$ is a system of parameters of $M$ such that $\underline{x} R$ is a minimal reduction of $\mathfrak{m}$. Since $\widetilde{M}$ is a Buchsbaum module by Theorem 1.1, we get

$$
J(\widetilde{M})=e(M)=e(\underline{x} ; M)=e(\underline{x} ; \widetilde{M}) \geq e(\widetilde{M}) \geq J(\widetilde{M}) .
$$

It follows that $e(\widetilde{M})=J(\widetilde{M})$. Therefore $\widetilde{M}$ is a Linear Maximal Buchsbaum $\hat{R}$ module and $\mu_{\widehat{R}}(\widetilde{M})=\sum_{i=0}^{d-1}\left(\begin{array}{c}d \\ i\end{array}\right) \ell\left(H_{\widehat{\mathfrak{m}}}^{i}(\widetilde{M})\right)$ by [17, Proposition 3.5].

(ii) $\Rightarrow$ (i) follows from Theorem 1.1 and the fact $e(\widetilde{M})=J(\widetilde{M})$. 
Acknowledgement. The authors would like to thank the referee for his useful suggestions.

\section{References}

[1] M. Auslander and D.A. Buchsbaum, Codimension and multiplicity, Ann. of Math., 68 (1958), 625-657.

[2] N.T. Cuong, On the least degree of polynomials bounding above the differences between lengths and multiplicities of certain systems of parameters in local rings, Nagoya Math. J., 125 (1992), 105-114.

[3] N.T. Cuong, N.T. Hoa and N.T.H. Loan, On certain length functions associated to a system of parameters in local rings, Vietnam J. Math., 27 (3) (1999), 259-272.

[4] N.T. Cuong, N.T. Hoa and L.T. Nhan, On modules whose local cohomology modules have generalized Cohen-Macaulay Matlis duals, East-West J. Math., 3 (2) (2001), 109--123.

[5] N.T. Cuong and V.T. Khoi, Modules whose local cohomology modules have CohenMacaulay Matlis duals, In Commutative Algebra, Algebraic Geometry, and Computational Methods (Editor D. Eisenbud), Spinger 1999, 223- 231.

[6] N.T. Cuong, M. Morales and L.T. Nhan, On the length of generalized fractions, J. Algebra, 265 (2003), 100-113.

[7] N.T. Cuong and N.D. Minh, Lengths of generalized fractions of modules having small polynomial type, Math. Proc. Cambridge Philos. Soc., 128 (2000), 269-282.

[8] N.T. Cuong and L.T. Nhan, Pseudo Cohen-Macaulay and pseudo generalized CohenMacaulay modules, J. Algebra, 267 (2003), 156-177.

[9] S. Goto, On the associated graded rings of parameter ideals in Buchsbaum rings, J. Algebra, 85 (1983), 490-534.

[10] S. Goto and K. Yamagishi, The theory of unconditioned strong $d$-sequences and modules of finite local cohomology, (Preprint, 1986).

[11] R. Hartshorne, Property of $A$-sequence, Bull. Soc. Math. France, 4 (1966), 61-66.

[12] J. Herzog, M.E. Kunz et al., Der kanonische Modul eines Cohen- Macaulay- Rings, Lecture Notes in Math., 238, Spinger-Verlag, Berlin-Heidelberg-New York, 1971.

[13] M. Hochster, Contraced ideals from integral extensions of regular rings, Nagoya Math. J., 51 (1973), 25-43.

[14] R.Y. Sharp and M.A. Hamieh, Lengths of certain generalized fractions, J. Pure Appl. Algebra, 38 (1985), 323-336.

[15] J. Stückrad and W. Vogel, Buchsbaum rings and applications, Spinger-Verlag, BerlinHeidelberg-New York, 1986.

[16] J.R. Strooker. Homological questions in local algebra, London Math. Lecture Notes Series, Vol. 145, Cambridge University Press, 1990.

[17] K. Yoshida, On linear maximal Buchsbaum modules and the syzygy modules, Comm. in Algebra, 23 (3) (1995), 1085-1130. 